\title{
Neck Radiotherapy Increases the Risk of Syncope During Awake Airway Management: A Report of Two Cases
}

\author{
Catherine Vu*, Gang Zheng and Carin Hagberg \\ Department of Anesthesiology and Perioperative Medicine, University of Texas MD Anderson Cancer Center, USA
}

Submission: July 23, 2019; Published:August 08, 2019

*Corresponding author: Catherine Vu, Department of Anesthesiology and Perioperative Medicine the University of Texas MD Anderson Cancer Center1400 Holcombe Blvd., Suite FC13.2007, Houston, TX 77030, USA

Abstract

Radiotherapy for head and neck cancer may causes baroreflex dysfunction and can lead to syncope. Syncope is usually a benign clinical condition and self-limit. However, in patients with difficult airway their spontaneous recovery can be interrupted by the significant hypoxia caused by airway obstruction. This might lead to situation of cannot intubate and cannot oxygenate requiring emergency surgical airway. Here we report two cases in which previous radiotherapy treated patients developed syncope during preparation for awake intubation resulting in emergency surgical access.

Keywords: Airway; Syncope; Neck radiotherapy; Awake airway management

Abbreviations: TLOC: Transient Loss of Consciousness; NRT: Radiotherapy to The Neck; BP: Blood Pressure; HR: Heart Rate; CICO: Cannot Intubate, Cannot Oxygenate; OR: Operating Room; BPM: Beats Per Minute; ETT: Endotracheal Tube; SCC: Squamous Cell Carcinoma; IV: Intravenous; LMA: Laryngeal Mask Airway

\section{Introduction}

Syncope is defined as Transient Loss of Consciousness (TLOC) owning to brain hypoperfusion. It is characterized by quick onset, short duration and is self-limited. It is generally a benign clinical condition and has a reported overall mortality rate of $0.28 \%$ from all causes [1]. Clinical reports have shown that Radiotherapy to The Neck (NRT) for cancer treatment causes impairment of the baroreflex response and results in baroreflex dysfunction. Studies have also demonstrated a positive correlation between NRT and syncope [2,3]. Although full compensation for baroreflex dysfunction may be achieved in daily life, these individuals may have a decreased tolerance to emotional or physical distress resulting in exacerbated Blood Pressure (BP) and Heart Rate (HR) changes with stimulation. We report two cases in which patients developed TLOC during airway preparation for awake intubation, resulting in the situation of "Cannot Intubate, Cannot Oxygenate (CICO)", and their airways were managed by emergency surgical access.

\section{Case 1}

62 -year-old, $78 \mathrm{~kg}$ female with a past medical history of controlled chronic hypertension and recurrent tongue cancer presented for direct laryngoscopy, tongue biopsy and possible tracheostomy. The patient underwent a partial glossectomy 13 months previously due to primary oral tongue cancer followed by bilateral neck radiotherapy. Tumor assessment revealed a large mass extending from the right base of the tongue to the ipsilateral vallecular region, (Figure 1) but no hypopharyngeal or laryngeal airway obstruction. Awake flexible scope intubation via nasal approach was planned. Upon arrival Operating Room (OR), the patient was placed on the OR table with the head elevated 30 degrees. Her first BP, HR and room air $\mathrm{O}_{2}$ saturation readings in the OR were $165 / 82 \mathrm{mmHg}, 71$ beats per minute (bpm) and 100\%, respectively. She received $2 \mathrm{mg}$ midazolam and $50 \mathrm{mcg}$ fentanyl intravenously upon arrival to the OR. Attempted placement of a \# 28 nasal trumpet into the nares for dilation purposes caused significant discomfort as the result 
of high resistance and difficult insertion, despite $4 \%$ lidocaine administration prior to instrumentation. She developed bradycardia and soon became unconsciousness with HR of 30s bpm. Her BP could not be obtained by sphygmomanometer. The patient was immediately placed spine and ventilated with $100 \%$ oxygen with a facemask. As the patient's HR and $\mathrm{O}_{2}$ saturation continued to deteriorate, $500 \mathrm{mcg}$ of epinephrine was titrated intravenously, while an emergent cricothyrotomy was performed with a $6 \mathrm{~cm}$ Endotracheal Tube (ETT) by the surgeon $\left(\mathrm{O}_{2}\right.$ saturation was in $\left.30 \mathrm{~s}\right)$. The patient's vital signs quickly recovered and the cricothyrotomy was then converted to a formal tracheotomy. The scheduled procedures were allowed to continue. The patient was allowed to regain consciousness after an uneventful surgical procedure. During the post-operative visit, the patient was queried, and she had no recollection of the intraoperative events. She was discharged from the hospital on post-operative day \#3. No further diagnostic tests were performed during the remainder of her hospitalization.

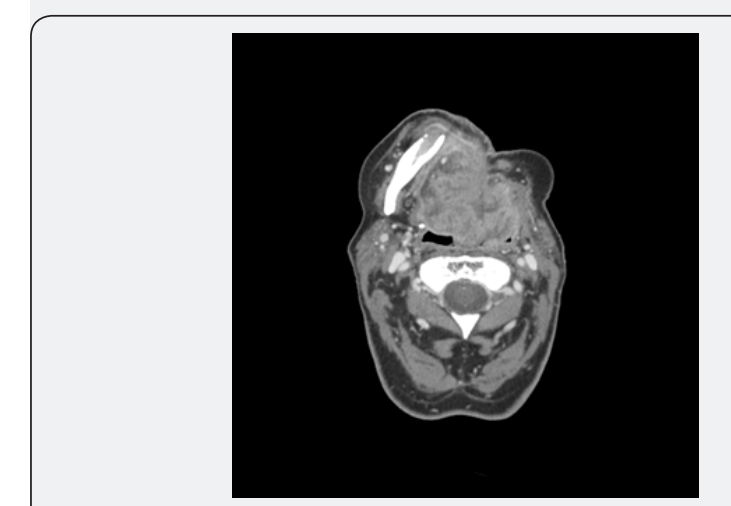

Figure 1: A large mass on axial plane of a CT imagine measured $74 \times 53 \mathrm{~mm}$.

\section{Case 2}

58-year-old female with a medical history of well-controlled chronic hypertension and right vocal fold recurrent Squamous Cell Carcinoma (SCC) presented for a total laryngectomy. Her primary laryngeal cancer was treated with bilateral neck radiotherapy two years prior to the surgery. Patient denied any history of cardiovascular disease. She presented with a weak and hoarse voice, but no difficulty breathing. She had a BMI of $38 \mathrm{~kg} / \mathrm{m} 2$ and neck circumference of $39 \mathrm{~cm}$. Airway assessment revealed a Mallampati 3 airway without significant abnormality in any other routine airway assessments. Video laryngoscopy performed one week prior to surgery revealed an exophytic tumor growing on her right vocal cord with immobilization of the ipsilateral true vocal folder. However, the airway was unobstructed. Based on the above findings, an awake flexible scope intubation was planned. The patient had a baseline BP, $\mathrm{HR}$, and oxygen saturation of 148/72mmHg, $82 \mathrm{bpm}$ and $98 \%$ on room air, respectively. In the $\mathrm{OR}$, the patient was placed in the siting position with $2 \mathrm{~L} / \mathrm{min}$ of $\mathrm{O}_{2}$ through a nasal cannula. While placing monitors, the patient became pale and then became unresponsive. The pulse oxygen waveform showed pulse rate of 30s bpm with weak pulse by palpation, but her BP could not be obtained by sphygmomanometer. Ephedrine (15mg) was immediately administered Intravenously (IV) followed by $200 \mathrm{mcg}$ of phenylephrine. Despite the efforts of mask ventilation with $100 \%$ oxygen, her $\mathrm{O}_{2}$ saturation continued falling. Trismus was also evident when attempting insertion of an oral airway. Subsequently, a Laryngeal Mask Airway (LMA) was placed after intravenous administration of $120 \mathrm{mg}$ succinylcholine, but no improvement of oxygenation was achieved. An emergent tracheostomy was performed at $\mathrm{O}_{2}$ saturation in the low $80 \mathrm{~s} \%$ by the surgeon. Thereafter, the patient was allowed to regain consciousness and exhibited a normal neurologic assessment. Surgery was then continued and completed in three hours. Postop 12 leads EKG showed a normal sinus rhythm with HR of $68 \mathrm{bpm}$. The cardiology consult suspected a vasovagal syncope as the cause of TOLC. No further evaluation was performed. In PACU visit, patient had no recollection of the intraoperative events. She underwent an uneventful hospitalization and was discharged from the hospital on post-operative day \#5.

\section{Discussion}

The sequential events developed in the two cases resembled a cycle of reflex syncope. In these two cases, previous NRT predisposed patients to potential baroreflex dysfunction. Emotional distress and/or additional painful stimulation played an important role in triggering the hemodynamic instability by exacerbated BP and HR changes resulting in TLOC. However, the self-recovery was interrupted by significant hypoxia caused by airway obstruction. Without quickly establishing invasive airway access, both cases may have resulted in significant patient morbidity.

In patients with reflex syncope, stimulation from the circulatory and visceral receptors to the brain via the corresponding somatic sensory pathway, glossopharyngeal or valgus nerves, strong emotional distress may directly activate or facilitate activation of this reflex. The efferent paths consist of the valgus nerve to heart and sympathetic fibers to the heart and vessels. The degrees of vasodepressor and bradycardia, or both, result in varying levels of manifestations. Most syncope attacks occur in the upright posture reflecting the mechanism of central hypovolemia in loss of consciousness and memory dysfunction during an attack [4]. In a healthy individual, the functional integrity of baroreflex plays a protective role in preventing syncope. Impaired baroreflex is a mechanism resulting in inability of sympathetic response to systemic hypotension $[5,6]$. A previous study demonstrated that impaired baroreflex predicts the development of syncope during tilted test [7].

Baroreflex failure resulting from NRT is a rare clinical entity. However, radiotherapy-induced baroreflex dysfunction is common. Lack of heart rate and blood pressure response to valsalva maneuverer and vasoactive challenging tests in patients undergone NRT were found in all the patients in the study [8,9]; 
and decreasing hemodynamic responses to surgical stimulations in patients with NRT undergoing general anesthesia was also reported [10]. Therefore, the patients with full compensation of dysfunctional baroreflex in their daily life may develop baroreflex decompensation under certain circumstances (the triggers) resulting in loss of hemodynamic autoregulation and episode of syncope.

Despite detrimental effects of TLOC developing at critical moments, a routine test of baroreflex function in asymptomatic patients is not practical. Therefore, carefully inquiry into the history of fainting or near fainting in patients who have undergone NRT is important to recognize the patients with increased risk of syncope. During airway management, emotional distress and anxiety should be controlled before the onset of an awake intubation procedure. Every effort should be made to prevent central hypoperfusion resulting in TLOC and collapse of airway by avoiding prolonged erect position and promptly treating hypotension and bradycardia. To manage a difficult airway, one must be aware that repeated stimulation of the glossopharyngeal nerve distribution (posterior $1 / 3$ of tongue) by airway instrumentation may worsen the symptoms and prolong the recovery period; therefore, the benefits versus the risk ratio of technique chosen to manage the airway must be carefully considered. Nevertheless, managing a difficult airway with a concurrent sudden onset event is challenging. Prevention and early treatment are key to success and the avoidance of catastrophic results.

\section{References}

1. Alshekhlee A, Shen WK, Mackall J, Chelimsky TC (2009) Incidence and mortality rates of syncope in the United States. Am J Med 122(2): 181188.
2. Timmers HJ, Karemaker JM, Lenders JW, Wieling W (1999) Baroreflex failure following radiation therapy for nasopharyngeal carcinoma. Clin Auton Res 9(6): 317-324.

3. Sharabi Y, Dendi R, Holmes C, Goldstein Ds (2003) Baroreflex failure as a late sequel of neck irradiation. Hypertension 42(1): 110-116.

4. Brignole M, Moya A, Lange F, Deharo J, Elliott P, et al. (2018) Practice Instruction for the 2018 ESC Guidelines for the diagnosis and management of syncope. Eur Heart J 39(21): e43-e80.

5. Mosqueda-Garcia R, Furlan R, Fernandez-Violante R, Desai T, Snell M, et al. (1997) Sympathetic and baroreceptor reflex in neutrally mediated syncope evoked by tilt. J Clin Invest 99(11): 2736-2744.

6. Jardine DL, Wieling W, Brignole M, Lenders JWM, Sutton R, et al. (2018) The pathophysiology of the vasovagal response. Heart Rhythm 15(6): 921-929.

7. Morillo CA, Eckberg DL, Ellenbogen KA, Beightol LA, Hoag JB, et al (1997) Vagal and sympathetic mechanisms in patients with orthostatic vasovagal syncope. Circulation 96(8): 2509-2513.

8. Timmers HJ, Karemaker JM, Wieling W, Kaanders JH, Folgering HT, et al. (2002) Arterial baroreflex and peripheral chemoreflex function after radiotherapy for laryngeal or pharyngeal cancer. Int J Radiat Oncol Biol Phys 53(5): 1203-1210.

9. Huang CC, Huang TL, Hsu HC, Chen HC, Lin HC, et al. (2013) Long-term effects of neck irradiation on cardiovascular autonomic function: a study in nasopharyngeal carcinoma patients after radiotherapy. Muscle Nerve 47(3): 344-350.

10. Zheng G, Dong W, Lewis CM (2017) General anesthesia imposes negative effects on heart rate and blood pressure regulation in patients with a history of head and neck radiation therapy. Anesth Analg 125(6): 2056-2062. 